\title{
Regular, narrow QRS, long RP tachycardia - what is the mechanism?
}

\section{S. Tzeis ${ }^{1}$ S. Pastromas ${ }^{1}$ A. Sikiotis ${ }^{1} \cdot$ G. Andrikopoulos ${ }^{1}$}

Published online: 16 September 2016

(C) The Author(s) 2016. This article is available at SpringerLink with Open Access.

We report the case of a 45-year-old male patient who was referred to our department due to palpitations for the last six months, with documented episodes of regular, long RP, narrow QRS tachycardias (Fig. 1). The patient had no history of structural heart disease, his transthoracic echocardiography demonstrated normal systolic and diastolic function and the thyroid function tests were within normal limits.
The 24-hour Holter monitoring showed multiple, recurrent, self-terminating episodes of regular, long RP tachycardias (Fig. 2).

The patient was referred for an electrophysiological study. During the procedure, the patient was almost continuously in a regular long-RP tachycardia. Any attempt of either atrial or ventricular pacing during intermittent
Fig. 1 Twelve-lead surface ECG of the index regular, long RP, narrow QRS tachycardia

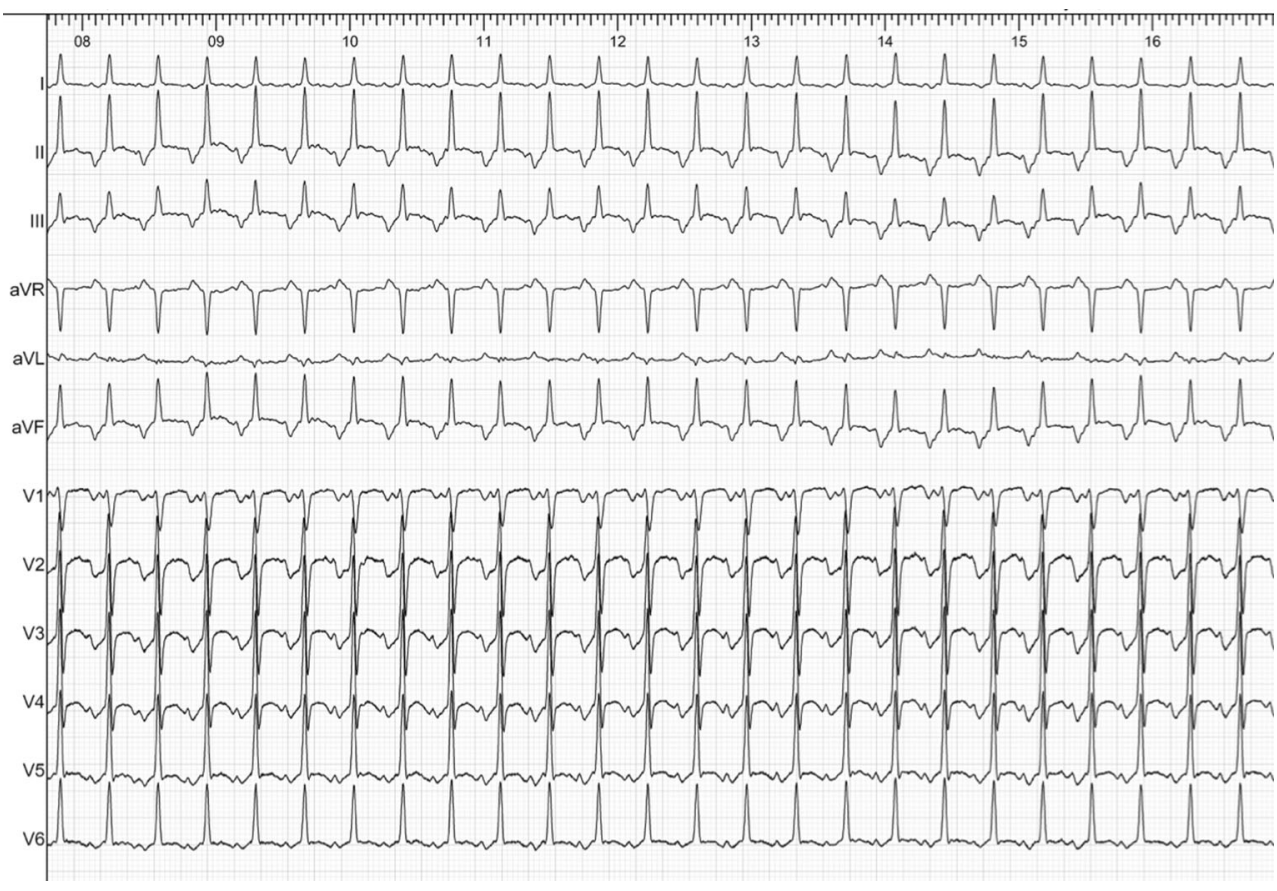

S. Tzeis

stzeis@otenet.gr

Pacing and Electrophysiology Department, Henry Dunant Hospital Center, Athens, Greece 
Fig. 2 Tracings from the Holter recording showing repetitive, self-terminating episodes of long-RP tachycardia. The horizontal arrows show the duration of the recurrent bouts of the tachycardia during rhythm recording

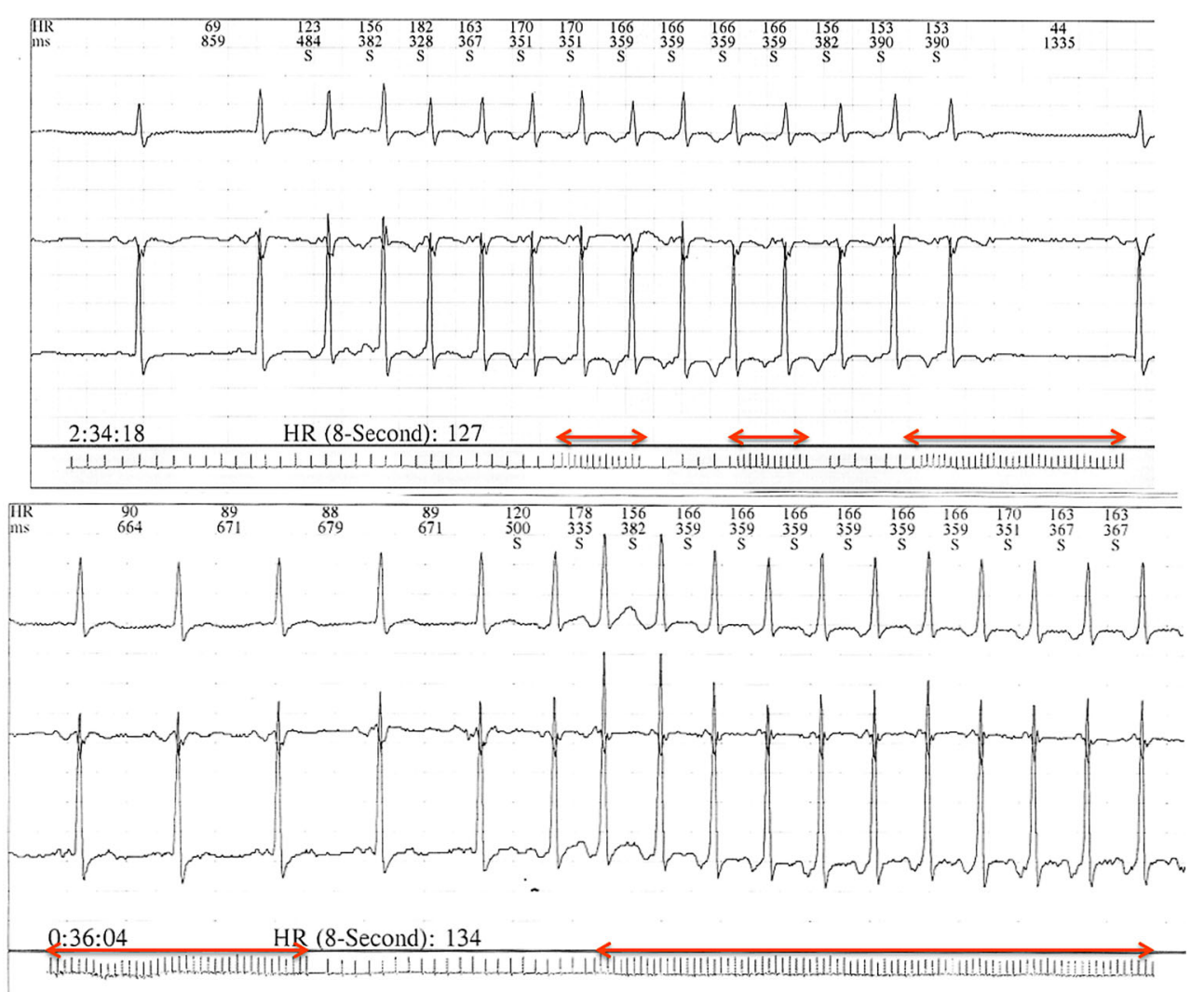

sinus rhythm resulted in reproducible tachycardia induction, which precluded the evaluation of the retrograde conduction properties. Therefore, only pacing manoeuvres during the index tachycardia could be used to differentiate the underlying mechanism.

What is the differential diagnosis of the index tachycardia?

Which diagnostic manoeuvre during the electrophysiological study is suggested for the delineation of the underlying tachycardia mechanism?

\section{Answer}

You will find the answer elsewhere in this issue.

Open Access This article is distributed under the terms of the Creative Commons Attribution 4.0 International License (http:// creativecommons.org/licenses/by/4.0/), which permits unrestricted use, distribution, and reproduction in any medium, provided you give appropriate credit to the original author(s) and the source, provide a link to the Creative Commons license, and indicate if changes were made. 